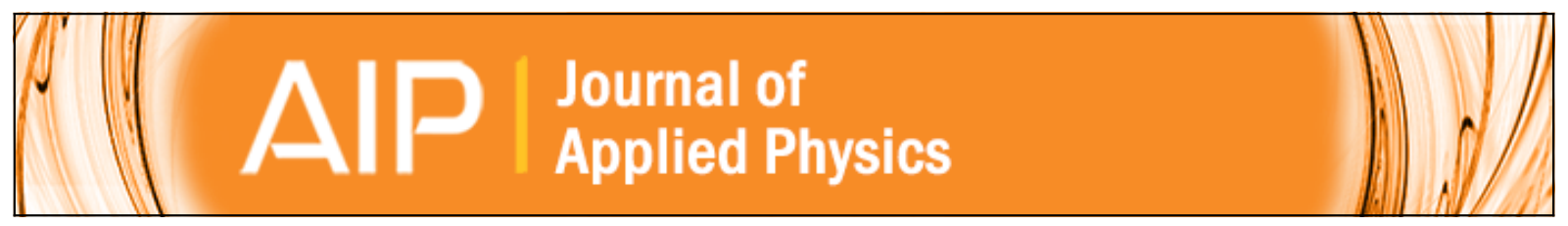

Influence of the postplasma process conditions on the surface conductivity of hydrogenated diamond surfaces

E. Snidero, D. Tromson, C. Mer, P. Bergonzo, John S. Foord, C. Nebel, Oliver A. Williams, and Richard B. Jackman

Citation: Journal of Applied Physics 93, 2700 (2003); doi: 10.1063/1.1539922

View online: http://dx.doi.org/10.1063/1.1539922

View Table of Contents: http://scitation.aip.org/content/aip/journal/jap/93/5?ver=pdfcov

Published by the AIP Publishing 


\title{
Influence of the postplasma process conditions on the surface conductivity of hydrogenated diamond surfaces
}

\author{
E. Snidero, ${ }^{\text {a) }}$ D. Tromson, C. Mer, and P. Bergonzo \\ LIST (CEA-Recherche Technologique)/DIMRI/SIAR, CEA/Saclay, 91191 Gif-sur-Yvette, France
}

John S. Foord

Physical and Theoretical Chemistry, University of Oxford, South Parks Road, Oxford, OX1 3QZ, United Kingdom

C. Nebel

Walter Schottky Institut, Technische Universität München, Am Coulombwall, D-85748 Garching, Germany

Oliver A. Williams and Richard B. Jackman

Electrical and Electronic Engineering, University College London, Torrington Place, London WC1E 7JE, United Kingdom

(Received 9 September 2002; accepted 3 December 2002)

\begin{abstract}
It is a common observation that diamond surface conductivity rises after exposure to hydrogen plasmas. Hydrogenation treatments are known to induce a $p$-type conductive layer, which is not present on non-hydrogenated samples. However, the particular mechanisms predominant in the plasma treatment process are still controversial, and several antagonist conditions have been reported to be of importance, such as sample temperature $\left(500{ }^{\circ} \mathrm{C}\right.$ to $800^{\circ} \mathrm{C}$ ), duration (a few seconds to $1 \mathrm{~h}$ ), and microwave (MW) power density, etc. Further, the post-plasma step is also crucial, especially since the surface conductivity has been reported to be affected by the presence of an adsorbate layer on the diamond surface. By setting up the arrangement to enable the in situ measurement of the surface conductivity after treatment, we have been able to control all parameters that could affect the surface conductivity, in order to determine those of importance. Among the parameters studied, we were able to analyze the influence of the surface temperature, the gas phase exposure (dry air, wet air, neutral gas, $\mathrm{CH}_{4}, \mathrm{O}_{2}$, and $\mathrm{H}_{2}$ ), the MW plasma conditions $\left(\mathrm{O}_{2}, \mathrm{H}_{2}\right)$ as well as the exposure to UV ( $\mathrm{Hg}$ and deuterium) and the importance of the sequence and duration of each of these treatments. We found that hydrogenated surfaces are strongly influenced by the combination of wet air exposure and UV light. We noticed that the effect of UV light is persistent and cannot be related to direct photoconduction and has to be attributed to a modification of the trapped defect population. This can, therefore, be compared with the modification of filled defect density as observed in persistent photoconduction. (c) 2003 American Institute of Physics.
\end{abstract}

[DOI: $10.1063 / 1.1539922]$

\section{INTRODUCTION}

It is a common observation that diamond surface conductivity rises after exposure to hydrogen plasmas. This conductivity is seen to persist for long periods under certain conditions, and has been reported to occur on single-crystal and polycrystalline diamond films grown by chemical vapor deposition (CVD) methods. ${ }^{1-3}$ Values of the surface conductivity in the range $10^{-5}$ to $10^{-3} \Omega^{-1} \mathrm{~cm}^{-1}$ are commonly observed, with the conductivity being due to the presence of holes; this $p$-type conductivity disappears after oxidation of the surface. ${ }^{2-14}$ Among the techniques that have been reported to create the H-surface layer, exposure to microwave $(2.45 \mathrm{GHz})$ stimulated hydrogen plasmas seems to be the most efficient technique. With a wide range of experimental conditions (pressure, temperature, and duration) appearing to stimulate the effect. The origin of this $p$-type conduction has been controversial with several models being discussed,

a) Author to whom correspondence should be addressed; electronic mail: esnidero@cea.fr principally the formation of a surface hole accumulation layer through the presence of adsorbates, ${ }^{4}$ the presence of shallow acceptor states in the near-surface region, ${ }^{13}$ and, most recently, the formation of a surface hole accumulation layer through the presence of either adsorbates or metals with an appropriate electronegativity. ${ }^{14}$ This form of $p$-type conductivity has been used to form field-effect transistors on single-crystal, ${ }^{15}$ highly oriented $\mathrm{CVD}^{16}$ and polycrystalline CVD diamond. ${ }^{17}$ Denisenko and co-workers have attempted to consider the origin of the conductivity in terms of device characteristics. ${ }^{18}$

Most recent observations conclude that although a hydrogen-terminated surface is required before this form of $p$-type conductive surface layer can be formed, alone, it is insufficient. For example, without removing such adsorbed hydrogen, the surface conductivity can be varied from around $10^{-11}$ to $10^{-3} \Omega^{-1} \mathrm{~cm}^{-1}$ by modification of the environmental conditions. ${ }^{10}$ Implicated factors are the initial surface condition, the presence of water or oxygen containing gases, thermal desorption of surface species, the presence 
of metallic layers and light promoted effects. Most early studies failed to isolate these processes; this article forms part of an on-going collaboration between the authors that aims to control each experimental parameter independently, leading to an enhanced insight into the origin and usefulness of this form of $p$-type conduction on diamond. In this study, we have measured surface conductivity in situ after exposure to a microwave-stimulated hydrogen plasma. A similar study has been carried out by Cannearts and co-workers. ${ }^{19}$ In this case, electrical connections were used that remained permanently in contact with the sample during all steps prior to and following plasma exposure. This approach leads to the exposure of the electrical probes to atomic hydrogen at high temperatures, and can, hence, result in their corrosion. As such, it is possible that re-evaporated electrode material may erroneously affect any electrical measurements made. Further, it is clear that the plasma itself could be altered if the sample surface is kept at a fixed bias or simply even connected to two electrical contacts that may act as microwave absorbers. Hence in the current study, removable electrical probes have been deployed throughout. This approach has also been previously taken by Foord et al. ${ }^{20}$ Only small changes in the insulating character of the diamond surface were observed when surfaces freshly hydrogenated in a microwave plasma were exposed in situ to water vapor or a range of common gases in separate experiments. However, a large increase in surface conductivity was seen if the surface was first exposed to water vapor and then other gasses including oxygen, carbon dioxide, and formic acid. Conductivity changes were also observed ex situ in solutions of differing $p \mathrm{H}$, although irretrievable loss of the conductive phase was observed if the diamond was heated in alkaline solutions.

\section{EXPERIMENTAL DETAILS}

Samples were mounted in a plasma chamber with a base pressure of $10^{-7} \mathrm{mbar}$, which has been described in detail elsewhere. ${ }^{21}$ The system uses $2.45 \mathrm{GHz}$ microwave energy to form a resonant standing wave within the vacuum region of the chamber and can cause the formation of an atomic hydrogen-based three-dimensional plasma "ball" upon filling of the chamber with hydrogen. Typical conditions used here were $1 \mathrm{~h}$ exposures to samples held at a surface temperature of $820^{\circ} \mathrm{C}$, using $50 \mathrm{mbar}$ pressure, $120 \mathrm{sccm}$ hydrogen flow, and $900 \mathrm{~W}$ power. Following this, samples were cooled in hydrogen gas at 50 mbar for $1 \mathrm{~h}$. A mechanical manipulator was used to enable two copper probes to be positioned on the sample surface for conductivity measurements, while allowing their removal during plasma treatments. Conductivity measurements were performed using a Keithley 6517A electrometer. For all results reported here, a bias supply of $5 \mathrm{~V}$ is used. The system was equipped with five gas lines for either plasma or postplasma exposures, using argon, oxygen, nitrogen, hydrogen, or methane. A resistive heater under the substrate holder enabled annealing treatments up to $800^{\circ} \mathrm{C}$ to be performed. In this way, the sample could be dehydrogenated and similar initial conditions obtained for new experiments without exposing the sample to air. A water cell connected to the reactor and kept

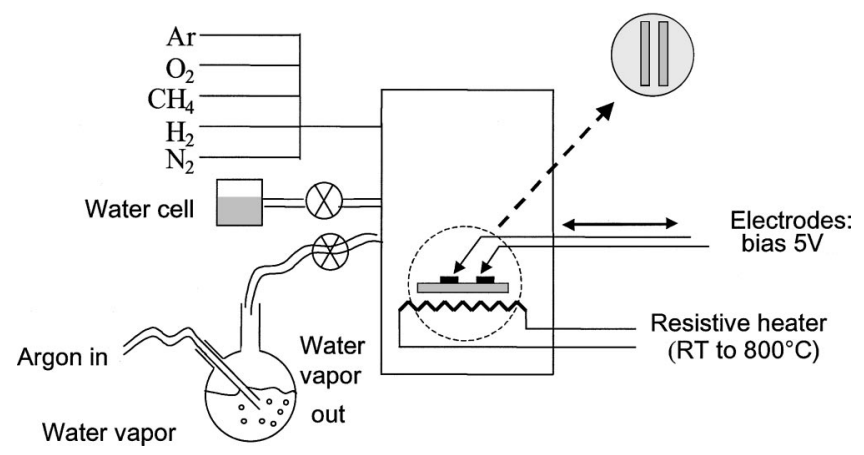

FIG. 1. Experimental setup.

at room temperature allowed a feed of gaseous water into the process chamber; this flow could also be assisted using an argon carrier gas. A schematic representation of the apparatus is shown in Fig. 1.

All the results reported here were obtained from the same free-standing polycrystalline diamond sample, with dimensions of $50 \mathrm{~mm}$ diameter and $64 \mu \mathrm{m}$ thickness. The sample displayed random grain orientations. Two large area metal electrodes $(40 \mathrm{~mm} \times 5 \mathrm{~mm})$, separated by a $1.5 \mathrm{~mm}$ gap, were deposited onto the sample surface before positioning in vacuo. The electrode material consisted of stacked layers of gold and platinum, which offered good resilience following hydrogen and oxygen plasma exposures.

\section{RESULTS}

The first set of experiments addressed the influence of dry gases on hydrogenated diamond surfaces. Directly after a hydrogen plasma treatment, the measuring probes were brought into contact with the sample, measuring a surface conductivity value of only $10^{-12} \Omega^{-1} \mathrm{~cm}^{-1}$. Dry argon was then fed into the chamber, and Fig. 2 shows the evolution of the surface conductivity that was measured with the argon partial pressure plotted on the same axis. These plots indicate little or no change in the conductivity is observed up to partial pressures as high as 800 mbar. Exactly the same experi-

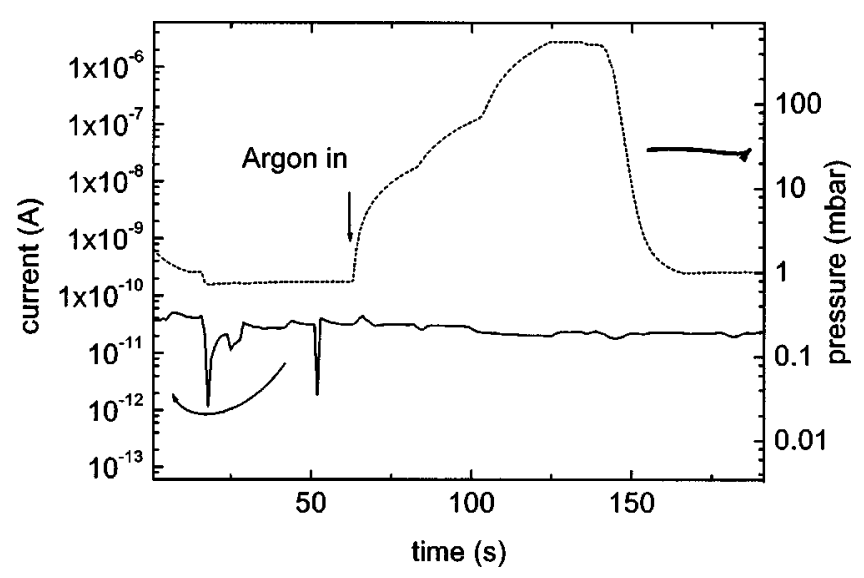

FIG. 2. Evolution of hydrogenated surface current after plasma treatment as the pressure is varied from vacuum 800 mbar with $\mathrm{Ar}, \mathrm{O}_{2}, \mathrm{~N}_{2}$, and $\mathrm{CH}_{4}$ $+\mathrm{N}_{2}$ gaseous mixtures. 


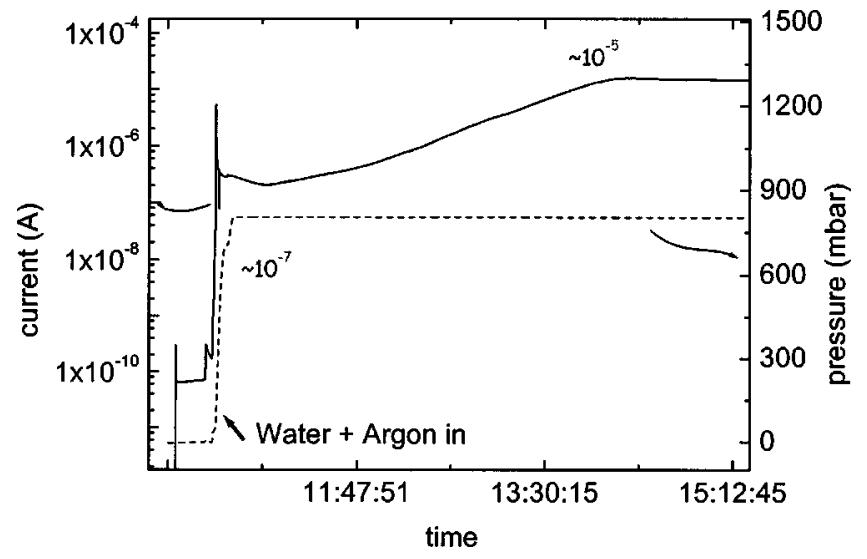

FIG. 3. Rise in the surface conductivity $\left(10^{-12}\right.$ to $\left.10^{-5} \Omega \mathrm{cm}^{-1}\right)$ after $\mathrm{H}_{2} \mathrm{O}$ exposure. The equilibrium is reached after $5 \mathrm{~h}$.

ment was then carried out with other dry gases, namely $\mathrm{O}_{2}$, $\mathrm{N}_{2}$, and a mixture of $\mathrm{N}_{2} / \mathrm{CH}_{4}$; the same result was obtained.

A second set of experiments involved exposing the hydrogenated diamond surface to water vapor before filling the chamber with dry gas. Initially, a water cell was used: When opened to the reactor, rapid pressure equilibrium was reached typically of around 20 mbar; the chamber was then increased in pressure using argon gas. A significant increase in surface conductivity is observed, reaching values of $10^{-8}$ $\Omega^{-1} \mathrm{~cm}^{-1}$. However, it is clear that this value is far from the values expected for the most conductive layers that have been reported in literature, where values higher than $10^{-5} \Omega^{-1} \mathrm{~cm}^{-1}$ are typically expected.

A third set of experiments aimed to exposure the hydrogenated diamond surfaces to higher partial pressures of water using a heated water source and, again, an argon carrier gas. The results of this experiment are plotted in Fig. 3, where, again, the current observed is plotted alongside the water/ argon partial pressure. In this case the rise in conductivity observed is of seven orders of magnitude from $10^{-12}$ to $10^{-5} \Omega^{-1} \mathrm{~cm}^{-1}$.

In these experiments the effect of UV light, from a nonfiltered $\mathrm{D}_{2}$ lamp, on the surface conductivity of hydrogenated samples was studied. In the preceding experiments, it has been shown that hydrogenated surfaces exposed to water vapor show conductivities that can increase by up to seven orders of magnitude as compared to dry surfaces. Water exposed hydrogenated diamond surfaces were used here, where following exposure of the chamber to 800 mbar of water/ argon, the diamond surface was exposed to UV light through a fused silica view port. Figure 4 shows the evolution of the conductivity: A further order of magnitude rise in the conductivity is observed. This progressive increase is extremely slow and takes affect over a couple of hours, with a measured light flux of approximately to $10^{13}$ photons $\mathrm{cm}^{-2} \mathrm{~s}^{-1}$. However, when UV exposure ceases, little decrease in the surface conductivity was observed. This persistent conductivity was extremely stable, lasting for weeks after exposure (Fig. 5). Similar observations were made when the wavelength of the light was varied above and just below the band gap energy of diamond $(5.5 \mathrm{eV}, 225 \mathrm{~nm})$, using filters on the

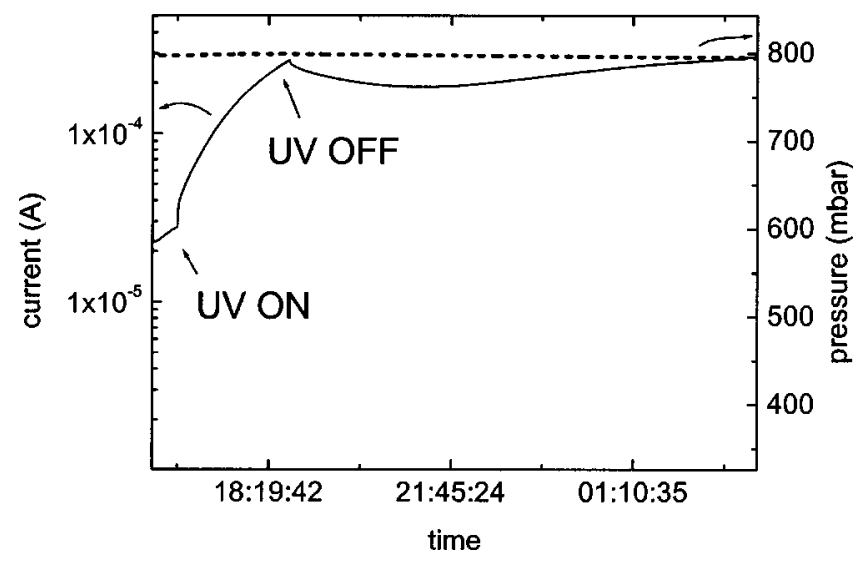

FIG. 4. Following the described exposure to water vapor, the surface is exposed to UV illumination. A rise in the conductivity is observed from $10^{-11}$ to $10^{-3} \Omega \mathrm{cm}^{-1}$.

$\mathrm{D}_{2}$ light source, or making use of an alternative mercury vapor lamp.

The effect of evacuating the chamber after this experiment was then investigated. The evolution of the sample conductivity observed is shown in Fig. 6. The pressure in the chamber is reduced during rough pumping, to the value at which water starts to evaporate significantly (typically $<30$ mbar). In this phase, very high levels of water vapor become available in the chamber, and a significant rise in conductivity was observed: From $0.25 \times 10^{-3}$ to $0.5 \times 10^{-3}$ $\Omega^{-1} \mathrm{~cm}^{-1}$. Then, when most of the water has been pumped from the chamber, the pressure was reduced further (turbomolecular pumping); the conductivity began to drop and was observed to do so irremediably for several days.

\section{DISCUSSION}

The most striking observation from the current study is that the combination of water exposure and UV light on hydrogenated surfaces gives rise to extreme surface conductivities of the order or $10^{-3} \mathrm{ohm}^{-1}$. This result conflicts with some previous reports, where nonwater based adsorbates were required before significant surface conduction could be

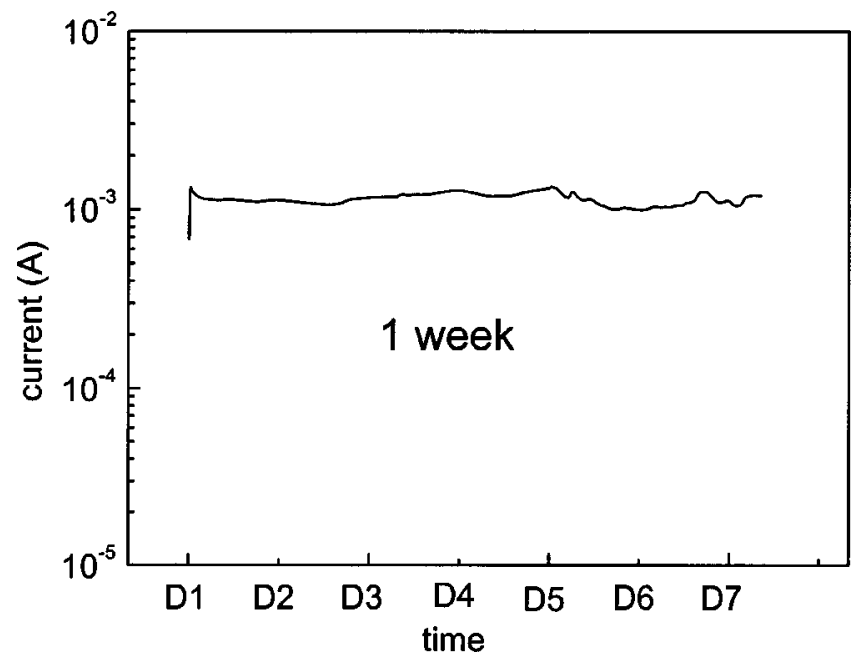

FIG. 5. Stability of the surface conductivity over one week in the dark. 


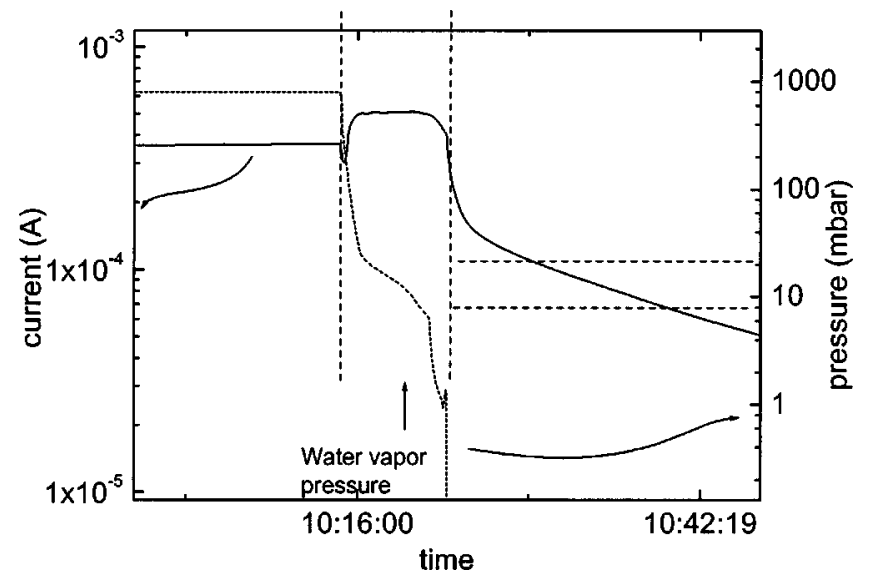

FIG. 6. Evacuating the water vapor from the chamber; between 20 and 6 mbar (below room-temperature water vapor pressure), the conductivity rises from $0.25 \times 10^{-3}$ to $0.5 \times 10^{-3} \Omega \mathrm{cm}^{-1}$. Below, the conductivity drops as the remaining water vapor is removed from the chamber.

seen. ${ }^{19,20}$ For example, in the work of Foord et al., ${ }^{20} \mathrm{CVD}$ diamond surfaces freshly hydrogenated in a microwave plasma were found to be insulating (as here), but even when exposed in situ to water vapor and other common atmospheric gases in individual exposures no conductivity was observed. However, exposure to water vapor followed by exposure to gases such as formic acid, oxygen, or carbon dioxide produced a large increase in conductivity, although nitrogen gas was found to be ineffective in this regard. These results were qualitatively consistent with previous suggestions that the conductivity at exposed hydrogenated diamond surfaces arises through electron transfer to adsorbates, which is mediated by the presence of a "wetting layer." It is, therefore, possible that in the present case, low levels of impurities have been fed into the chamber with the argon gas in which case our observations would be consistent with this report. However, when the system was exposed to argon (plus any potential impurities) alone, no rise in conductivity was observed (initial experiment). Conversely, exposure to saturated water vapor without argon carrier gas did provoke high levels of surface conduction, although not as high as that achieved with higher water vapor pressures carried with argon gas. Since the effective distillation of water into the plasma chamber is unlikely to be accompanied by significant impurity gases it can be stated that water alone can provoke high levels of surface conductivity, but that a wider range of adsorbates may be implicated in the formation of surfaces which display the highest possible levels of electrical conduction. In this case, the present study is consistent with previous observations, but uniquely indicates the vital role of water or waterlike adsorbates in the promotion of diamond surface conductivity.

The second most significant observation from the work performed here is the clear demonstration that exposure of water saturated surfaces to UV light leads to a further rise in surface conductivity, even though the exact photon energy (above and below the band gap energy) does not seem to play a significant role. The photon fluxes used here do not lead to photoconductivity at the conduction levels observed; the light must provoke an enhancement of the hydrogenated diamond surface conductivity. UV light modification of the wet surface properties, that can take one of two forms: (i) activation of water species on the diamond surface, or (ii) the modification of diamond electrical properties when exposed to UV. CVD diamond is known to support a large number of defect states that could be activated by the UV light. Techniques such as thermoluminescence, thermally stimulated currents, and deep level transient spectroscopy have identified trapping levels localized around $1.2 \mathrm{eV}$ within the band gap which can be emptied at elevated temperatures of $550 \mathrm{~K}$. In the same manner that the trapped population on these levels affects the radiation detection properties of diamond, it is likely that it also modifies the surface conductivity of the hydrogenated surface. In fact, if the surface conductivity relies on the formation of an adsorbate layer to which electrons have to be provided from the diamond, it is likely that the modification of the trapped populations in the diamond bulk will vary this ability of the diamond to provide these electrons to the surface layer.

To clarify this, we have conducted a final experiment. After annealing $\left(400^{\circ} \mathrm{C}\right)$, the surface conductivity dropped to values of the order of $\mathrm{pA}$, level of the lower limit of the measurement setup, this value corresponding to those measured just before the hydrogenation process and after the oxygen plasma treatment. At this point, the sample surface was then exposed to UV light before being exposed to water. We observed that water exposure then allows one to directly reach the high levels of surface conductivity as observed when UV was shined after water exposure. This demonstrates that the UVs do not play a significant effect on modifying or activating water species on the surface, but rather helps promote the creation of the conductive layer by modifying the trapped level population in the diamond.

The order of importance of UV or water exposure does not, therefore, play a significant role, and since the hydrogen plasma are intense sources of UVs, we can realize here how the temperature of the diamond at which the plasma is switched off becomes of importance. If the plasma is extinguished below the temperature at which levels are emptied (typically $500 \mathrm{~K}$ ), then the defect levels remain populated, therefore rendering the adsorbate layer formation easier and enabling higher levels of conductivity. One other way, therefore, is to shine UVs on the sample before or after exposure to water.

\section{CONCLUDING REMARKS}

All these investigations on the nature of the hydrogenated surface aspire to identify the parameter(s) responsible for the rise of the surface conductivity after an hydrogen plasma treatment in order to obtain a reproducible protocol to control the quality of the hydrogenation. We have seen that water alone seems to have a significant contribution on the diamond surface conductivity. Further, the effect of light and particularly in the UV domain gives evidence that the stability of the surface conductivity may be partly governed also by the status of the trapped population of deep level defects. Since this trapped population can be affected by external factors like light and heat, care has to be taken when 
the sample is brought back to atmosphere after the hydrogen plasma treatment. The inherent stability of devices based on the surface conductivity of diamond must imply that the surface properties remain stable and kept away from other sources of gas as well of light as a crucial step for device fabrication.

\section{ACKNOWLEDGMENTS}

The authors would like to thank all the components of the DoDDs project (Doped Diamond Devices and Sensors) RTN-HPRN-CT-1999-00139 supported by the European Commission.

${ }^{1}$ M. I. Landstrass and K. V. Ravi, Appl. Phys. Lett. 55, 975 (1989); 55, 1391 (1989)

${ }^{2}$ H. Kawarada, M. Aoki, H. Sasaki, and K. Tsugawa, Diamond Relat. Mater. 57, 718 (1994).

${ }^{3}$ H. J. Looi, J. S. Foord, and R. B. Jackman, Appl. Phys. Lett. 72, 353 (1998).

${ }^{4}$ R. S. Gi, T. Mizumasa, Y. Akiba, Y. Hirose, T. Kurosu, and M. Iida, Jpn. J. Appl. Phys., Part 1 34, 5550 (1995).

${ }^{5}$ M. Itoh and H. Kawarada, Jpn. J. Appl. Phys., Part 1 34, 4677 (1995).

${ }^{6}$ B. L. Mackey, J. N. Russel Jr., J. E. Crowell, and J. E. Butler, Phys. Rev. B 52, 17009 (1995).

${ }^{7}$ H. J. Looi, L. Y. S. Pang, A. B. Molley, F. Jones, J. S. Foord, and R. B. Jackman, Diamond Relat. Mater. 7, 550 (1998).
${ }^{8}$ H. J. Looi, L. Y. S. Pang, M. D. Whitfield, J. S. Foord, and R. B. Jackman, Thin Solid Films 344, 623 (1999).

${ }^{9}$ J. B. Cui, J. Ristein, and L. Ley, Diamond Relat. Mater. 9, 1036 (2000).

${ }^{10}$ J. Ristein, F. Maier, M. Riedel, M. Stammer, and L. Ley, Diamond Relat. Mater. 10, 416 (2000).

${ }^{11}$ B. Mantel, M. Stammer, J. Ristein, and L. Ley, Diamond Relat. Mater. 10, 429 (2001).

${ }^{12}$ O. A. Williams, M. D. Whitfield, R. B. Jackman, J. S. Foord, J. E. Butler, and C. E. Nebel, Diamond Relat. Mater. 10, 423 (2001).

${ }^{13}$ K. Hayashi, S. Yamanaka, H. Okushi, and K. Kajimura, Appl. Phys. Lett. 68, 376 (1996).

${ }^{14}$ C. E. Nebel, F. Ertl, C. Saurer, M. Stutzmann, C. F. O. Graeff, P. Bergonzo, O. A. Williams, and R. B. Jackman, Diamond Relat. Mater. 11, 351 (2002).

${ }^{15}$ A. Hokazono, T. Ishikura, K. Nakamura, S. Yamashita, and H. Kawarada, Diamond Relat. Mater. 6, 339 (1997).

${ }^{16}$ P. Gluche, A. Aleksov, A. Vescan, E. Ebert, and E. Kohn, IEEE Electron Device Lett. 18, 547 (1997)

${ }^{17}$ H. J. Looi, L. Y. S. Pang, Y. Wang, M. D. Whitfield, and R. B. Jackman, IEEE Electron Device Lett. 19, 112 (1998).

${ }^{18}$ A. Denisenko, A. Aleksov, A. Tribil, P. Gluche, W. Ebert, and E. Kohn, Diamond Relat. Mater. 9, 1138 (2000).

${ }^{19}$ M. Cannearts, M. Nesladek, K. Haenen, L. De Schepper, and C. Van Haresendonck, Phys. Status Solidi A 186, 235 (2001).

${ }^{20}$ J. S. Foord, C. H. Lau, M. Hiramatsu, R. B. Jackman, C. E. Nebel, and P. Bergonzo, Diamond Relat. Mater. (to be published).

${ }^{21}$ D. Tromson, P. Bergonzo, A. Brambilla, C. Mer, F. Foulon, and V. Amosov, J. Appl. Phys. 87, 3360 (2000). 